ARTICLE

\title{
Ph2 encodes the mismatch repair protein MSH7-3D that inhibits wheat homoeologous recombination
}

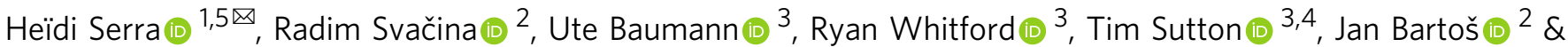 \\ Pierre Sourdille (iD) ${ }^{1 \times}$
}

Meiotic recombination is a critical process for plant breeding, as it creates novel allele combinations that can be exploited for crop improvement. In wheat, a complex allohexaploid that has a diploid-like behaviour, meiotic recombination between homoeologous or alien chromosomes is suppressed through the action of several loci. Here, we report positional cloning of Pairing homoeologous 2 (Ph2) and functional validation of the wheat DNA mismatch repair protein MSH7-3D as a key inhibitor of homoeologous recombination, thus solving a half-century-old question. Similar to ph2 mutant phenotype, we show that mutating MSH7$3 \mathrm{D}$ induces a substantial increase in homoeologous recombination (up to 5.5 fold) in wheatwild relative hybrids, which is also associated with a reduction in homologous recombination. These data reveal a role for MSH7-3D in meiotic stabilisation of allopolyploidy and provides an opportunity to improve wheat's genetic diversity through alien gene introgression, a major bottleneck facing crop improvement.

\footnotetext{
${ }^{1}$ Genetics, Diversity and Ecophysiology of Cereals, UMR 1095, INRAE, Université Clermont Auvergne, Clermont-Ferrand, France. ${ }^{2}$ Institute of Experimental Botany of the Czech Academy of Sciences, Centre of the Region Hana for Biotechnological and Agricultural Research, Olomouc, Czech Republic. ${ }^{3}$ School of Agriculture, Food and Wine, University of Adelaide, Waite Campus, PMB1, Glen Osmond, SA, Australia. ${ }^{4}$ South Australian Research and Development Institute, Adelaide, SA, Australia. ${ }^{5}$ Present address: Genetics, Reproduction and Development, CNRS, Inserm, Université Clermont Auvergne, Clermont-

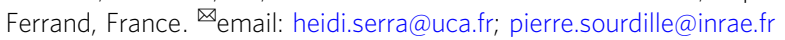


C rop wild relatives provide a valuable source of genes and allelic variants for abiotic stress tolerance, disease resistance and quality traits that are important for breeding, particularly in the context of human population growth and a changing climate. Remarkable progress has been made over the last 80 years, with notable boosts in the 1970s and 1980s ${ }^{1}$, in knowledge and resulting methodology to allow utilisation of wild relatives in wheat breeding. However, an important challenge still facing breeders now is the ability to routinely perform DNAintrogression, a process by which distantly related chromosomes exchange genetic information that is passed onto progeny. The transfer of chromatin between pairing maternal and paternal chromosomes relies on recombination, a process which occurs in all sexually reproducing species during meiosis ${ }^{2}$.

The genetics of chromosome pairing and meiotic recombination is complicated by the allopolyploid nature of many crops, a widespread feature in the plant kingdom ${ }^{3}$. For example, hexaploid bread wheat (Triticum aestivum L., AABBDD $2 n=6 x=$ 42), which derives from two successive interspecific crosses involving three diploids ${ }^{4,5}$, has three sets of related homoeologous chromosomes. Genetic and cytogenetic studies have revealed the presence of several pairing homoeologous $(P h)$ loci that ensure wheat behaves as a diploid during meiosis, with only homologous chromosomes of the same sub-genome (AA, BB or $\mathrm{DD}$ ) pairing and recombining. The two main loci controlling homoeologous recombination are located on chromosome-arms 5BL and 3DS, named $P h 1$ and $P h 2$, respectively ${ }^{6-9}$.

Analysis of $P h 1$ gene mutants in tetraploid $(p h 1 c)^{10,11}$ and hexaploid $(p h 1 b)^{12}$ first identified interstitial deletions involving an $\sim 0.84-\mu \mathrm{m}$ region and a $1.05-\mu \mathrm{m}$ region around the gene, respectively ${ }^{13,14}$. Subsequent physical mapping localised $P h 1$ to a $2.5 \mathrm{Mbp}$ region on chromosome $5 \mathrm{BL}^{15}$. This region contains a meiotic gene ZIP4 and a heterochromatin tandem repeat block, inserted within a cluster of CDK2-like genes ${ }^{15-18}$. ZIP4 is a ZMM protein involved in homologous recombination and may act as a hub through physical interactions with components of the chromosome axis and other $\mathrm{ZMMs}^{19}$. Recent evidence now points to TaZIP4-B2 (the additional ZIP4 copy on 5BL) as being responsible for the effect of this locus on homoeologous recombination ${ }^{20,21}$. Although the exact mode of action is unknown, TaZIP4-B2 seems to act as a focal point, facilitating physical interactions between components of the chromosome axis and crossover machinery ${ }^{17}$.

In comparison to $P h 1$, the causative gene sequence for $P h 2$ is yet to be determined. Analysis of the irradiation-mutant $p h 2 a$ in comparison to the syntenic region on rice chromosome 1 estimated the deletion to be at least $80 \mathrm{Mb}$ in size ${ }^{22}$, but more likely to span a 120 to $125 \mathrm{Mb}$ region ${ }^{23}$ on the terminal portion of 3DS. Research aiming to identify $P h 2$ has resulted in the isolation of a number of candidate meiotic genes from this region on 3DS. These include the genes WM124,25,WM3 $3^{26}, W M 5^{27}$ and TaMSH $7^{28,29}$. Despite these attempts, the region and its candidate meiotic gene content was deemed too large and complex to confidently identify the $P h 2$ causative sequence using the $p h 2 a$ deletion mutant alone. The chemically induced $p h 2 b$ mutant ${ }^{30}$, thought to contain either a point mutation or small lesion at $P h 2$, offered the prospect of identifying the causative gene sequence.

Identifying the genetic control and underlying mechanism of action of Ph 2 would provide valuable knowledge, and enable novel resources to be developed for introgressing alien sequences from related species into bread wheat. The use of ph2 mutation could be of particular interest to breeders and geneticists as it induces only a minimal disruption to endogenous homologous recombination $^{12,31,32}$ but reinforces phlb's effect of promoting homoeologous recombination in some crosses ${ }^{33}$.
Here, we report the positional cloning of $\mathrm{Ph} 2$ from a $121.16 \mathrm{Mb}$ candidate region on 3DS. Based on the analysis of a set of specifically created 3DS deletion mutants ${ }^{23}$ combined with exome sequencing and transcriptome analysis of $p h 2 a$ and $p h 2 b$ mutants versus wild-type, we identify TaMSH7-3D, a gene encoding a plant specific DNA mismatch repair protein. Using independent ethyl methanesulfonate (EMS) generated $m s h 7-3 D$ mutants crossed with wheat wild relative Aegilops variabilis, we demonstrate that $m s h 7-3 D$ mutants recapitulate the ph2 phenotype with a highly significant (5.5-fold) increase in homoeologous recombination and a reduction in homologous recombination. These data suggest that, in addition to TaZIP4-B2, TaMSH7-3D is an attractive target for facilitating alien gene introgression in prebreeding and breeding programs.

\section{Results}

Molecular characterisation of $p \boldsymbol{h} \mathbf{2} \boldsymbol{a}$ and $p \boldsymbol{h} \mathbf{2 b}$ mutations. The $\mathrm{Ph} 2$ pairing homoeologous locus is located on chromosome-arm 3DS within the terminally deleted region of irradiation-mutant ph $2 a^{9,31}$. Marker-based analysis of ph $2 a$ recently revealed that minimum deletion size is $120 \mathrm{Mb}$ with a maximum of $125 \mathrm{Mb}^{23}$. To precisely delineate the deletion breakpoint, ph $2 a$ was genotyped using a high-density SNP genotyping array (35 K SNP Affymetrix Axiom ${ }^{\circledast}$ ). Genotyping data showed the deletion breakpoint on chromosome 3D located between markers AX178057815 and AX-178057206 at the coordinates 120.722.379 and 121.539.725, respectively (Supplementary Fig. 1a). This was further refined using exome capture of ph $2 a$ : the deletion breakpoint is around the coordinate 121.163.000, upstream of the gene Traes3D01G153800 (Supplementary Fig. 1b). Using the newly available Chinese Spring Reference Genome v1.0 ${ }^{34}$, we identified 1577 genes within the deleted ph $2 a$ region.

To identify possible candidate genes for $P h 2$, we performed an exome capture of the EMS induced ph $2 b$ mutant, in which a point mutation was proposed as being responsible for the observed phenotype ${ }^{30}$. Comparison between $p h 2 b$ exome sequence and the Chinese Spring reference genome highlighted 165 single nucleotide differences within the $121.16 \mathrm{Mb}$ deleted $p h 2 a$ region. These consisted mainly of $\mathrm{G}$ to $\mathrm{A}$ and $\mathrm{C}$ to $\mathrm{T}$ transitions, as would be expected from alkylation by EMS treatment. We detected 59 SNPs within genic regions (including $5^{\prime}$ and $3^{\prime}$ UTR and potential promoter regions), among these 36 were exonic mutations (13 synonymous, 21 non-synonymous and 2 non-sense mutations) and one likely to affect transcript splicing (Supplementary Table 1). Considering only those genes that contain exonic SNPs predicted to result in either non-synonymous amino acid changes, protein truncations (premature STOP codons) or alternate splicing, the total number of $P h 2$ candidate genes was reduced to 24 .

Ph2 locates within a 14.3-Mb region on 3DS. Since these 24 candidate genes were dispersed over the entire length of the chromosomal region deleted in $p h 2 a$, we then sought to delineate Ph2 spatially. With this purpose, we developed a series of 113 wheat deletion lines carrying terminal deletions of chromosome $3 \mathrm{D}^{23}$. Among these lines, a subset of 32 that possessed 3DS deletions ranging in size from 6.5 to $142.6 \mathrm{Mb}$ were selected. The region between each adjacent deletion breakpoint of the tiled series did not exceed 14.3 Mb. Since the mutant phenotype for $P h 2$ is easily discernible in ABDR haploid hybrids ${ }^{35}$, each selected deletion line (in 3D monosomic constitution) was crossed with rye $(\mathrm{RR}, 2 n=14)$ and hybrids carrying a mutant $3 \mathrm{D}$ chromosome (terminal deletion) were selected in the progeny using $3 \mathrm{D}$ specific markers. We screened for the presence of $P h 2$ by characterising 


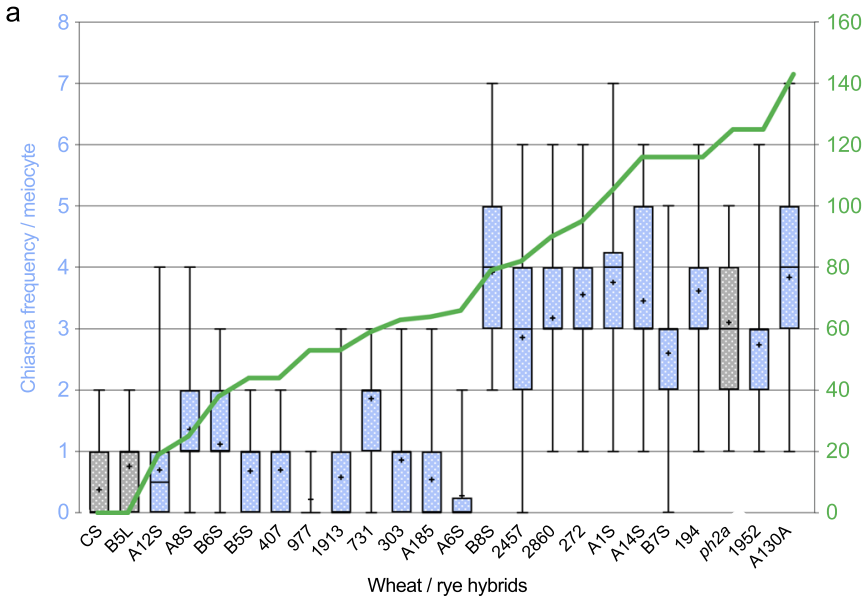

$\mathrm{b}$

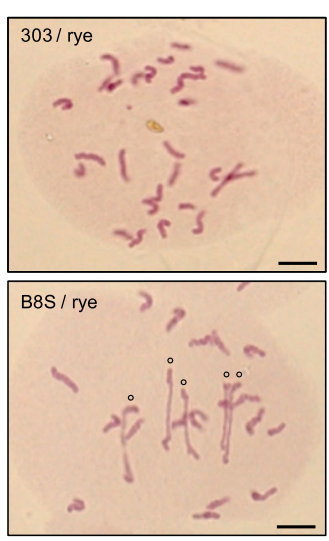

Fig. 1 Physical mapping of $\mathbf{P h 2}$ using wheat/rye hybrids carrying 3DS terminal deletions. Meiotic phenotypes at metaphase I of 24 wheat/rye hybrids were analysed cytogenetically. This analysis includes three wheat/rye hybrid controls (in grey) derived from: wild-type wheat cv. Chinese Spring (CS), CS carrying a $357 \mathrm{Mb}$ deletion of 3DL but no 3DS deletion (B5L) and CS ph2a mutant (ph2a). a Box plots showing minimum, first quantile, median (horizontal middle line), third quantile and maximum count of chiasma frequency per meiocyte for each hybrid. 50 meiocytes have been examined by hybrid and the mean values are represented by crosses. The green line indicates length (in Mb) of the 3DS terminal deletion carried by each hybrid. $\mathbf{b}$ Representative meiocytes at metaphase I of '303/rye' and 'B8S/rye' haploid hybrids showing chromosome configurations. '303/rye' meiocyte exhibits 28 univalents while 'B8S/rye' shows 18 univalents and 5 rod bivalents (o). Scale bars represent $10 \mu \mathrm{m}$ for both panels. Source data underlying Fig. 1a are provided as a Source Data file.

meiotic behaviour at metaphase I of 22 ABDR hybrids (each carrying a $3 \mathrm{D}$ chromosome with a terminal deletion). While haploid sets of wheat and rye homoeologous chromosomes rarely associated in wild-type hybrids $(0.38 \pm 0.10$ chiasma/meiocyte $)$, mean chiasma frequency was significantly increased in the ph $2 a$ mutant context $(3.10 \pm 0.13$ chiasmata/meiocyte $)$, indicating that formation of chiasmata between non homologous chromosomes occurs more frequently in the absence of Ph2 (Fig. 1; Supplementary Table 2$)^{31}$. Cytogenetic analyses of the generated wheat 3D-deletion line/rye hybrids revealed that individuals carrying a 3DS terminal deletion of $79.2 \mathrm{Mb}$ or more exhibit a high chiasma frequency (ranging from 2.60 to 3.92 chiasmata/meiocyte), similar to that observed for ph2a (Fig. 1; Supplementary Table 2). However, hybrids carrying a 3DS terminal deletion shorter than 64.9 $\mathrm{Mb}$ (minimal breakage position of the A6S line) showed less than 2 chiasmata/meiocyte indicating the presence of $\mathrm{Ph} 2$ and its ability to inhibit homoeologous recombination within these lines. Taken together, these data clearly demonstrate that $P h 2$ is located within a $14.3 \mathrm{Mb}$ genetic interval ranging from 64.9 to $79.2 \mathrm{Mb}$ on chromosome-arm 3DS.

TaMSH7-3D is a unique candidate for Ph2. Among the 24 candidate genes identified by $p h 2 b$ exome sequencing, only one located between positions 64.9 and $79.2 \mathrm{Mb}$ on 3DS. This gene, TraesCS3D02G119400, contains 17 exons and 16 introns with a total length of $9747 \mathrm{bp}$ and encodes the DNA mismatch repair protein TaMSH7-3D (Fig. 2). In ph2b, a G to A transition was detected at position 74.359 .312 and confirmed by Sanger sequencing. It affects the first nucleotide of the splicing pattern GTAAGT at the junction between exon 5 and intron 5 and is predicted to compromise correct splicing of the transcript. No other unique mutation from ph $2 b$ derived sequences was detected for this gene's A or B homoeologues (TraesCS3A02G117500 and TraesCS3B02G136600, respectively) nor for previously identified potential candidates for $P h 2$ : the WM1 gene family (TraesCS3D02G034300, TraesCS3D02G034500, TraesCS3D02G034700, TraesCS3D02G034900, TraesCS3D02G0 35200, TraesCS3D02G035100) 24,25, WM3 (TraesCS3D02G152900) ${ }^{26}$ or WM5 (TraesCS3D02G140300) ${ }^{27}$. We were unable to confirm the presence of three previously identified SNPs in the TaMSH7-3D coding sequence of $p h 2 b$, none of which were deemed to result in a non-functional or malfunctioning protein ${ }^{29}$. To determine whether the G to A SNP we identified here affects intron-5 splicing (leading to a predicted loss of protein function and therefore the $p h 2$ phenotype), we performed a high-depth RNAseq from wild-type and ph $2 b$ mutant anthers staged from pre-meiotic interphase to metaphase I. RNAseq data from $p h 2 b$ confirmed the presence of the splice junction mutation and showed that this mutation leads to the use of a downstream splice site. This results in a frame-shift and thereby creates a premature in-frame STOP codon (Supplementary Fig. 2). This STOP codon is predicted to result in a truncated, non-functional TaMSH7-3D protein missing major functional domains, specifically the core domain and the C-terminal ATPase domain (Fig. 2). Taken together, deletion-line mapping combined with exome and transcriptome sequencing of the $p h 2 b$ mutant identifies TaMSH7-3D as a unique candidate for $\mathrm{Ph} 2$.

Validation of TaMSH7-3D as the causative gene for Ph2. To functionally validate that TaMSH7-3D affects homoeologous recombination, we took advantage of the Targeting Induced Local Lesions In Genome (TILLING) population of 1200 wheat mutant lines of the variety Cadenza and the corresponding databases cataloguing mutations identified through exome sequencing (www.wheat-tilling.com) ${ }^{36,37}$. Screening by BLAST search identified 127 possible mutants for TaMSH7-3D (Traes_3DS_72259 A292.1) within the population. We selected four mutant lines with either a high probability of being knocked-out (Tamsh7-3D Q605*; stop codon gained; data described hereafter) or carrying missense mutations (Supplementary Table 3, Supplementary Figs. 3 and 4; data described in supplementary information). Considering the wheat variety Cadenza does not produce viable F1's when crossed with rye, the selected TaMSH7-3D mutants, as well as a wild-type Cadenza (Cad wt), were crossed with the wheat wild relative Aegilops variabilis (UUSS, $2 n=4 x=28$ ). The frequencies of univalents, rod and ring bivalents as well as multivalents were scored at meiotic metaphase I in the resulting F1 wheat/Ae. variabilis hybrids and were used to calculate total chiasma frequency per cell. Cad wt/Ae. variabilis hybrids exhibited on average $32.79( \pm 0.18)$ univalents and $1.10( \pm 0.09)$ rod 


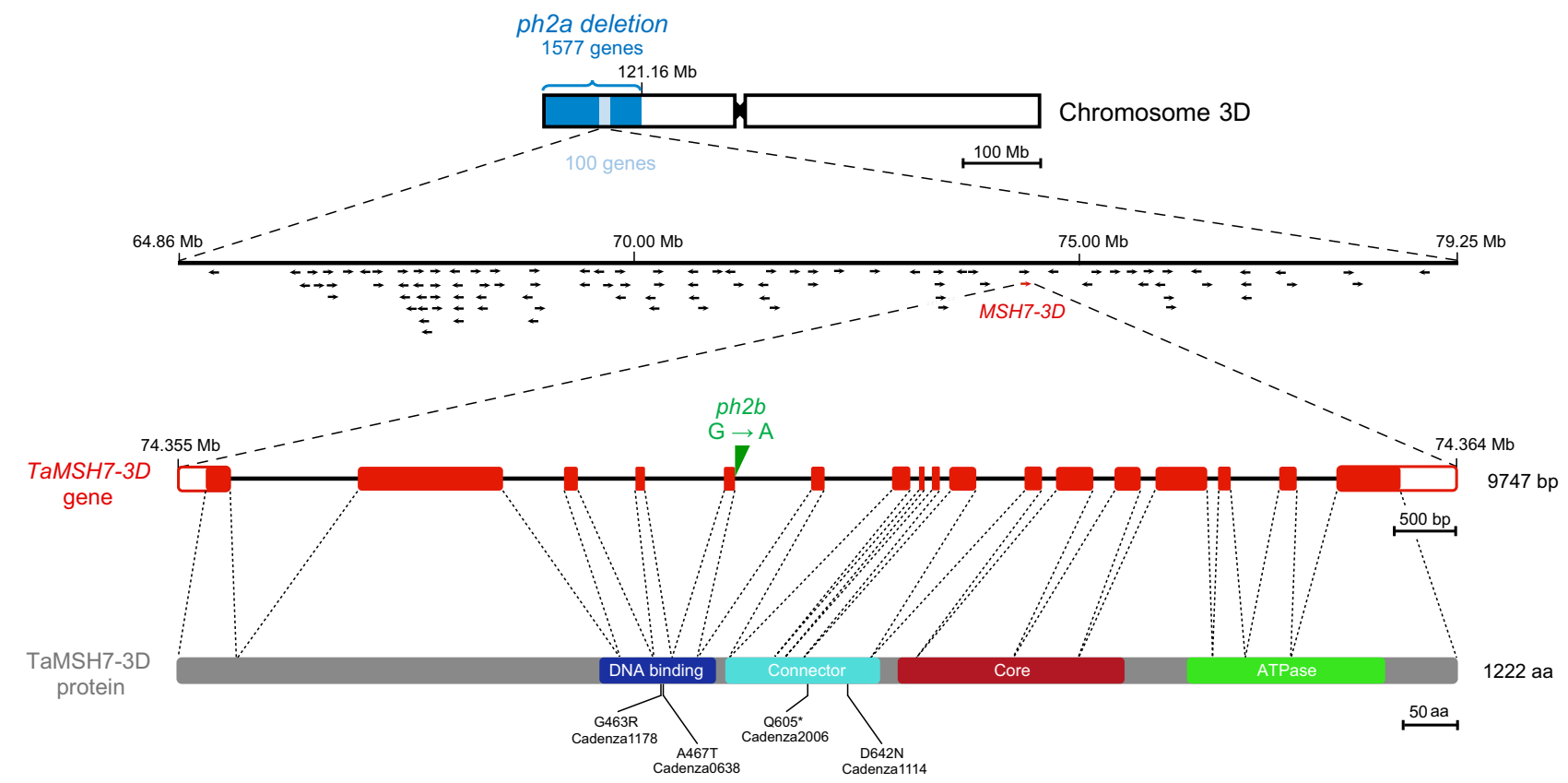

Fig. 2 Positional cloning of Ph2 identified TaMSH7-3D as the causative agent. Schematic representation of chromosome 3D showing ph2a deletion (dark blue). Further deletion-line mapping localised Ph2 to a $14.3 \mathrm{Mb}$ genetic interval (light blue) containing 100 genes (represented by arrows). Among them, the only gene identified to contain an exonic SNP predicted to either result in a non-synonymous amino acid (aa) change, protein truncation or alternate splicing in ph2b is TaMSH7-3D (TraesCS3D02G119400) at the coordinates 74.355.077 - 74.364.823 (highlighted in red). The G to A transition at position 74.359 .312 in ph $2 b$ sequence is shown (in green) in the gene structural schematic for TaMSH7-3D. TaMSH7-3D contains 17 exons (red rectangles) and 16 introns (black lines) with a total length of 9747 bp. 5' and 3' UTR's are represented by white rectangles. The schematic representation of the TaMSH7-3D protein is shown below. Regions encoding predicted protein domains are highlighted by coloured rectangles: $\mathrm{N}$-terminal mismatch-recognition domain (aa 405-515), connector domain (aa 525-672), core domain (aa 689-905) and C-terminal ATPase domain (aa 967-1154) containing a Walker A motif. Thin vertical lines below indicate positions of the aa changes in the four TILLING Tamsh7-3D mutants used in this study and * represents stop mutation.

bivalents corresponding to a mean chiasma frequency of $1.10( \pm$ $0.09)$ per meiocyte at metaphase I (Supplementary Table 4). In contrast, Tamsh7-3D Q605*/Ae. variabilis hybrids exhibited a 5fold increase in bivalent number $(5.29 \pm 0.15 \mathrm{rod}$ and $0.26 \pm 0.04$ ring bivalents per meiocyte on average) and the presence of multivalents $(0.12 \pm 0.03)$ (Fig. 3a, b; Supplementary Table 4). This is associated with a highly significant 5.52 -fold increase in mean chiasma frequency $(6.07 \pm 0.17$; Mann-Whitney test, $p=$ $7.43 \times 10^{-42}$ ) (Fig. 3c; Supplementary Table 4). The absence of a full-length TaMSH7-3D protein thus induces a substantial increase in genome-wide homoeologous recombination within the hybrid context. This data clearly demonstrates that TaMSH7$3 \mathrm{D}$ inhibits recombination between homoeologous chromosomes.

Moreover, a previous study reported a slight reduction in homologous recombination efficiency in wheat in the absence of $P h 2^{32}$. We first confirmed that chiasma frequency is significantly reduced in $p h 2 b$ relative to Chinese Spring wild-type $(37.48 \pm 0.26$ versus $40.98 \pm 0.14$, respectively; Mann-Whitney test, $p=3.04 \times$ $10^{-15}$ ) due to an observed increase in univalent and rod bivalent frequencies for $p h 2 b$ (Fig. 3e, f; Supplementary Table 5). As expected, Tamsh7-3D Q605* exhibited a similar phenotype (mean chiasma frequency of $37.58 \pm 0.19$ ) (Fig. 3d-f; Supplementary Table 5). Interestingly, rare trivalents and quadrivalents were also observed in $p h 2 b$ and Tamsh7-3D Q605* meiocytes (but not in those of a wild-type background), revealing that homoeologous recombination occurs in wheat in the absence of Ph2/TaMSH7-3D, albeit in the presence of Ph1. To test for TaMSH7-3D dosage sensitivity, we quantified chiasma frequency when only one functional copy of TaMSH7-3D is present (i.e. Tamsh-3D Q605* heterozygote) relative to two. Heterozygous plants showed an intermediate phenotype between wild-type and Tamsh-3D Q605* homozygotes (Supplementary Table 5,
Supplementary Fig. 5), indicative of a dosage dependent effect of TaMSH7-3D on homologous recombination.

Comparison of all genes containing EMS-derived SNPs in Tamsh7-3D Q605* and in ph $2 b$ identified 16 genes carrying mutations in both lines (Supplementary Table 6). Among these 16 genes, only MSH7-3D is located on 3DS (where Ph2 gene function was previously shown to be present based on ph $2 a$ analysis $^{31}$ ) and none of the 15 other genes carry mutation in ph $2 a$. These data eliminate the possibility of another gene contributing to the ph 2 phenotype.

Taken together, these data demonstrate that TaMSH7-3D lossof-function mutants have the capacity to recapitulate the ph2 phenotype with a substantial increase in homoeologous recombination in wheat/Ae. variabilis hybrids and a slight reduction of homologous recombination in wheat. These findings reveal a key role for TaMSH7-3D in inhibiting recombination between homoeologous chromosomes and consequently, in assuring accurate chromosome segregation during meiosis.

Tamsh7-3D reduces pollen viability but does not affect plant fertility. To assess whether meiotic behaviour disorders caused by Tamsh7-3D were associated with changes in fertility, we performed Alexander staining of pollen and scored the proportion of viable versus non-viable grains. Compared to wild-type, Tamsh7$3 D$ Q605* showed a slightly higher proportion of non-viable pollen $\left(p=6 \times 10^{-6}\right.$, pairwise $t$-test with correction for multiple testing) (Supplementary Fig. 6, Supplementary Table 7). We also measured seed-set and observed that seed number per spike in Tamsh7-3D Q605* was not significantly reduced compared to wild-type ( $p=0.43$, pairwise $t$-test with correction for multiple testing) (Supplementary Fig. 6, Supplementary Table 8). These 
a

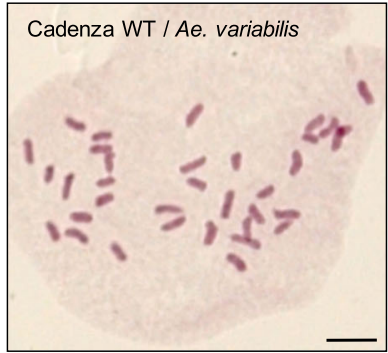

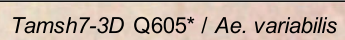

Tamsh7-3D Q605*/ Ae. variabilis

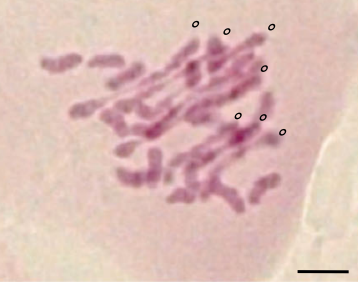

d
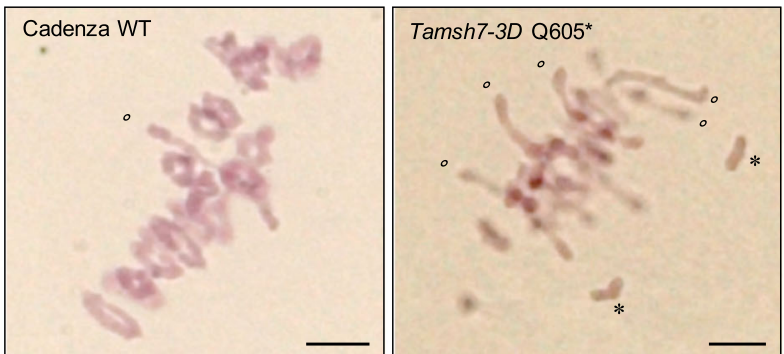

b

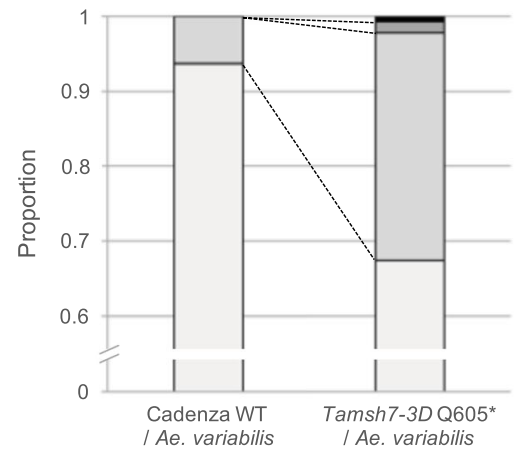

- Multivalents

Ring bivalents

Rod bivalents

Univalent pairs

e

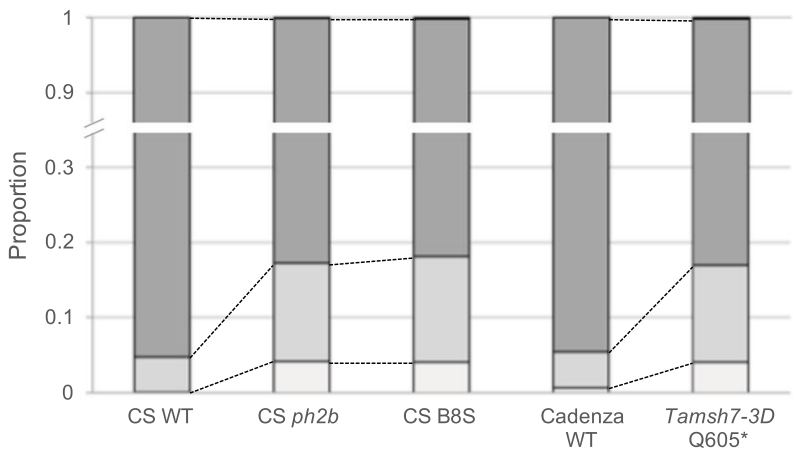

$f$

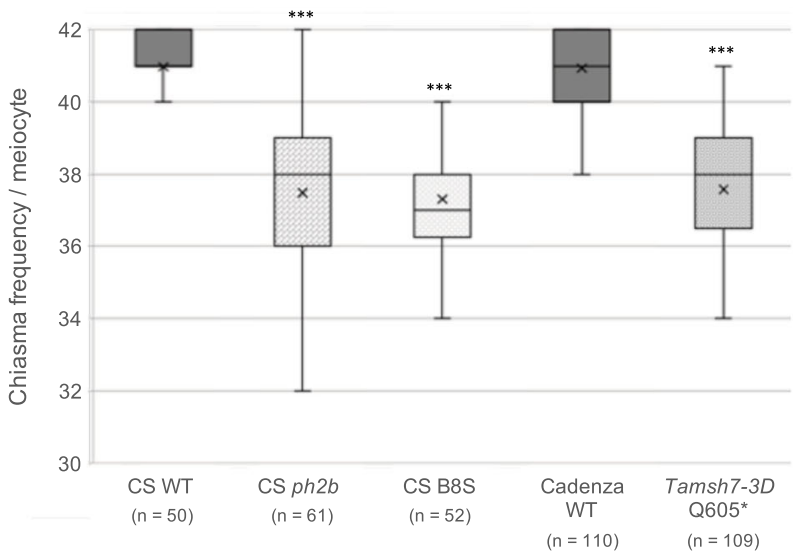

Fig. 3 Loss-of-function TaMSH7-3D mutations promote homoeologous recombination and reduce homologous recombination. a, b, c Meiotic chromosome phenotype at metaphase I of wheat cv. Cadenza/Aegilops variabilis haploid hybrids. Hybrids have 35 homoeologous chromosomes and the presence of bivalents and/or multivalents at metaphase I are therefore markers of homoeologous recombination (i.e. crossovers established between homoeologous chromosomes). d, e, f Meiotic chromosome phenotype at metaphase I of wheat cv. Chinese Spring (CS) or Cadenza. In a wild-type (WT) context, most homologous chromosome pairs are connected by two distal crossovers that form ring bivalents at metaphase I. The presence of rod bivalents and/or univalents reveals a reduction in homologous recombination efficiency. a, d Chromosome configurations of representative meiocytes at metaphase I. Open circles and asterisks indicate rod bivalents and univalents, respectively. Scale bars represent $10 \mu \mathrm{m}$ for all panels. b, e Stacked bar graphs showing mean proportions of each metaphase I chromosome configuration (univalent pairs, rod and ring bivalents, multivalents). c, $\mathbf{f}$ Box plots showing minimum, first quantile, median (horizontal middle line), third quantile and maximum count of chiasma frequency per meiocyte. Mean values are represented by crosses. $n$ number of cells examined. To test for differences between each mutant and corresponding wild-type control, two-sided Mann-Whitney tests adjusted for multiple comparisons were performed. ${ }^{\star \star \star}$ reports a $p$ value $<0.0001$ (see Supplementary Tables 4 and 5 for exact $p$ values). Source data underlying Fig. $3 c$ and $3 f$ are provided as a Source Data file.

data demonstrate that TaMSH7-3D loss-of-function does not significantly affect wheat fertility (as comparable seed sets are observed in the mutants) although this mutation does disturb proper homologous recombination (and induces homoeologous recombination events in some meiocytes). These results are in agreement with studies of tomato and Arabidopsis, in which reduced MSH7 expression or MSH7 loss-of-function (respectively) do not affect seed number ${ }^{38,39}$.

TaMSH7-3D is expressed in anthers during meiotic prophase $\mathrm{I}$. To precisely determine TaMSH7-3D expression over the course of early meiosis, transcript-profiling using a sub-staged meiotic time series was performed on whole-wheat anthers. Four meiotic stages were analysed: late leptotene, zygotene/pachytene, diplotene/diakinesis and metaphase I. RNA-seq data revealed that TaMSH7-3D (as well as TaMSH7-3A and $3 B$ homoeologues) is expressed for the entirety of prophase, which is in agreement with a role of TaMSH7-3D in control of homoeologous recombination at meiotic prophase I (Fig. 4). TaMSH7-3D expression however, is not restricted to meiosis considering similar transcript abundance was detected for each of the $3 \mathrm{~A}, 3 \mathrm{~B}$ and $3 \mathrm{D}$ homoeologous copies in leaf, root and stem before flowering (Fig. 4$)^{40}$. To investigate whether an absence of the pairing homoeologous gene Phl could 


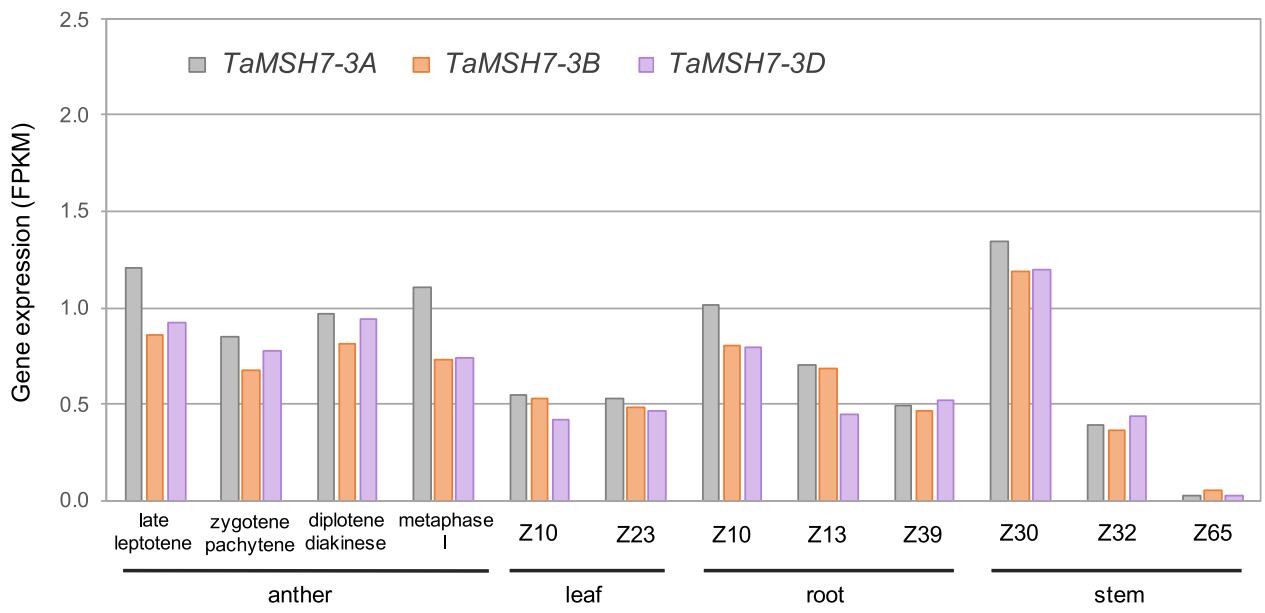

Fig. 4 TaMSH7-3A, TaMSH7-3B and TaMSH7-3D genes are expressed in anthers at early meiosis and in somatic tissues. Relative expression (in FPKM: Fragments per kilo base of transcript per million mapped reads) of the three TaMSH7 homoeologues in wild-type wheat anthers, leaves, roots and stems at various developmental stages. Stages according to Zadoks scale ${ }^{69}$ : Z10, seedling; Z13: three leaves unfolded; Z23, main shoot and three tillers; Z30, pseudostem erection; Z32, two nodes; Z39, flag leaf ligule and collar visible, Z65, half of flowering complete. Gene expression in anthers was obtained following the method described by Lloyd et al. ${ }^{59}$ and data is available at http://wheat-urgi.versailles.inra.fr/Seq-Repository/Expression. Gene expression in leaves, roots and stems is from Pingault et al. ${ }^{70}$.

be compensated by an overexpression of $P h 2 / T a M S H 7-3 D$, we compared TaMSH7-3D expression in wild-type versus the $p h 1 b$ mutant background using RNA-seq data previously generated ${ }^{41}$. No significant change in TaMSH7-3D transcript abundance (or TaMSH7-3A and $3 B$ ) was observed between wild-type and ph1b anthers at prophase I (Supplementary Fig. 7). This data indicates that the absence of $P h 1$ does not feedback to cause significant changes in TaMSH7 expression during meiosis in wheat.

TaMSH7-3D is more highly conserved than $3 \mathrm{~A}$ and $3 \mathrm{~B}$ homoeologues both among wild and domesticated wheats. Comparison of TaMSH7-3D with its homoeologous copies revealed they share more than $97 \%$ sequence identity at the nucleotide sequence level, with TaMSH7-3A being equidistant to TaMSH7-3B and TaMSH7-3D. This is reflected in their deduced protein sequences, TaMSH7-3B and $3 \mathrm{D}$ are $96.3 \%$ identical to TaMSH7-3A and $97.2 \%$ identical to each other (Supplementary Table 9). About $25 \%$ of amino acid differences between the homoeologues are concentrated within the region from amino acid 760 to 880 , thus in the MutS domain III corresponding to the core domain of the proteins (Supplementary Fig. 8).

Exome sequencing data revealed a high level of conservation of TaMSH7-3D among 436 bread wheat accessions studied. Only two haplotypes exist with the three identified SNPs exclusively localised within introns (Supplementary Table 10). In contrast, TaMSH7-3A and $3 B$ genes (inclusive of promoter regions) are more diverse with 12 and 30 polymorphisms identified within this population, respectively (Supplementary Table 10). By data mining NCBI's small read archive, querying the $10+$ Genome Project data and $\mathrm{DAWN}^{42}$, we identified 14 accessions that contain a 28 bp deletion in TaMSH7-3A, likely leading to a nonfunctional truncated protein (Supplementary Fig. 9, Supplementary Table 11). Pedigree analysis allowed us to deduce the most likely ancestor as Red-Fife, from which the deletion was transmitted (Supplementary Fig. 10). Considering only polymorphisms located within exons, we calculated the number of variants found in the 436 wheat lines within each gene and reported it relative to exon length. $13.69,6.74$ and 0 variants per $10 \mathrm{~kb}$ of exons were found in TaMSH7-3A, TaMSH7-3B and TaMSH7-3D, respectively. Consistent with the low genetic diversity of the D-genome, TaMSH7-3D is very well conserved within this collection. The TaMSH7-3A and TaMSH7-3B copies are however highly polymorphic: TaMSH7-3A sits within the top $30 \%$ of most diverse A genome derived genes and TaMSH7-3B within the top $20 \%$ of most diverse B-genome derived genes. Taken together, these data demonstrate that Ph2/TaMSH7-3D is more highly conserved than its homoeologues among wild and domesticated wheats, consistent with a major role for this gene in homoeologous recombination inhibition.

MSH7 is also highly conserved more broadly amongst the grasses. Bread wheat MSH7 proteins indeed show more than 70\% amino acid identity with MSH7 homologues of all studied Poaceae species (Supplementary Fig. 11, Supplementary Table 12). Protein sequence alignment of MSH7 homologues revealed that the main functional domains of the protein (MutS domain I, II, III and V) are particularly well conserved although the remaining regions of the protein display lower level of amino acid identity across species (Supplementary Fig. 12). This observation is in agreement with MSH7 function also being required for genome stability in more distantly related species.

\section{Discussion}

By 1952, it had become clear that corresponding bread wheat chromosomes derived from each subgenome were genetically very closely related, as observed through tetrasomy and nullisomy ${ }^{43}$. However the inability of these chromosomes to recombine during meiosis remained a paradox until a role for genetic suppressors was highlighted ${ }^{44}$. In this study, we report on the identification and functional validation of the key homoeologous chromosome pairing suppressor $\mathrm{Ph} 2$, through a combination of high-throughput exome and transcriptome sequencing of known mutants ( $p h 2 a$ and $p h 2 b$ ), cytogenetic analyses of both a 3DS deletion line series and independent EMS-induced mutants. We demonstrate that: (1) Ph2 locates within a $14.3-\mathrm{Mb}$ region ranging from 64.9 to $79.2 \mathrm{Mb}$ on 3DS; (2) TaMSH7-3D is the only gene localised within this region that contains an EMS-derived SNP susceptible to affect protein sequence in $p h 2 b$; (3) an additional mutant of TaMSH7-3D recapitulates the $p h 2$ phenotype in regards to homologous and homoeologous recombination; and (4) we were able to exclude all previously proposed candidates for 
Ph2 (not localised within the $14.3-\mathrm{Mb}$ newly refined $P h 2$ locus and not mutated in $p h 2 b$ ) except for TaMSH7-3D which had been fortuitously identified. Complementation of Tamsh7-3D mutants with a functional copy of the gene with its endogenous promoter would provide a direct evidence of this conclusion but plant regeneration after stable transformation remains recalcitrant in the currently available mutant backgrounds (Chinese Spring and Cadenza). Taken together, the provided data indicates TaMSH7$3 D$ as being the causative gene for $P h 2$, thus contributing to the resolution of a half-a-century-old question.

TaMSH7 (MutS homolog 7) is a plant specific member of the DNA mismatch repair (MMR) family. These highly conserved proteins play an essential role in maintaining genome stability by assuring the initial step of the MMR pathway, i.e. recognition of base-base mismatches and insertion/deletion mispairs generated during DNA replication and recombination ${ }^{45}$. MSH7 forms a heterodimer with $\mathrm{MSH} 2$ and the protein complex allows specific recognition of single-base mismatches including G/G, G/A, A/A and $\mathrm{C} / \mathrm{A}$ mispairs and to a lesser extent G/T, as shown by biochemical studies of Arabidopsis MSH2-MSH7 (MutSy) complex $^{46-48}$. The two heterodimeric complexes MSH2-MSH3 (MutS $\beta$ ) and MSH2-MSH6 (MutS $\alpha$ ), present in yeast, animals and plants, have different mismatch recognition properties and abilities to support MMR. MSH2-MSH3 senses large (2-16 nucleotides) insertion/deletion loops and interstrand crosslinks, whereas MSH2-MSH6 recognises single-base mismatches, including oxidative mispairs (dihydro-8-oxoguanine), methylated mispairs ( $\mathrm{O}^{6} \mathrm{meG}: \mathrm{T}$ and $\left.\mathrm{O}^{6} \mathrm{meG}: \mathrm{C}\right)$ and small (1-2 nucleotides) insertion/deletion loops ${ }^{49,50}$. MSH7 appears to have arisen early in plant evolution, most likely via duplication and divergence from a MSH6-like gene present in a primitive plant ${ }^{46,51}$, with this extra DNA lesion recognition protein likely contributing to efficient repair of various DNA damage caused by constant environmental exposure, for which plants are naturally subjected ${ }^{48}$.

We show that the absence of a functional TaMSH7-3D induces a 5.5-fold genome-wide increase in chiasma frequency in a bread wheat/Ae. variabilis hybrid context (Fig. 3a, c), providing evidence that this protein acts as a key inhibitor of homoeologous recombination. This finding is in line with a previous study assessing how frequently alien chromatin of wild tomato (Solanum lycopersicoides) is introgressed into cultivated forms (Solanum lycopersicum) following MSH7 silencing ${ }^{38}$. This study demonstrated a modest yet significant increase of $16.1 \%$ in recombination rate between these divergent chromosomes. In Arabidopsis, loss of AtMSH7 ( $m s h 7$ T-DNA insertion line) was observed to increase meiotic homologous recombination rate by $97 \%$ relative to wild-type at the subtelomeric 420 genetic interval as assessed using a fluorescent seed reporter line ${ }^{52}$. This data contrasts with a slight but significant reduction in genome-wide homologous recombination frequency observed for Tamsh7-3D in wheat (Fig. $3 \mathrm{~d}-\mathrm{f})^{32}$. In some Tamsh7-3D/ph2 wheat meiocytes, such a reduction in homologous recombination is associated with the presence of rare multivalents resulting from homoeologous recombination (Fig. 3e; Supplementary Table 5) ${ }^{31}$. This observation suggests that in a wild-type context, TaMSH7-3D plays a role in recombination partner choice (homologous vs homoeologous) likely through promoting destabilization of recombination intermediates established between homoeologous chromosomes. These intermediates could be less stable than those established between homologous sequences because of the presence of mismatches. A role for MMR proteins in recognising mismatches created in heteroduplex DNA (following DNAstrand exchange) and promoting dissociation of invading strand DNA - a process known as heteroduplex rejection - has indeed been reported ${ }^{53}$. In rice, MSH7 interacts with MEICA, an orthologue of FLIP known to be a partner of FIGL1 ${ }^{54}$. FIGL1/
FLIP is a conserved complex that regulates the strand invasion step of meiotic recombination ${ }^{55}$. Direct interaction between these two proteins is thus consistent with a role for TaMSH7-3D during this critical step. By preventing divergent DNA sequences from recombining, TaMSH7-3D would play a crucial role in assuring the diploid-like meiotic behaviour of polyploid bread wheat required for accurate chromosome segregation during meiosis. In diploid species, MSH7 may also be involved in limiting ectopic (non-allelic) recombination, a driver of highly deleterious chromosomal rearrangements, and could potentially provide an immediate advantage to newly formed allopolyploids by assuring meiotic stability and consequently, fertility.

Identification of the two main genes controlling homoeologous recombination in bread wheat, TaZIP4-B2 $2^{20}$ and TaMSH7-3D (this study), now offers a possibility of deciphering their direct mode of actions and interactions. Recent data from G. Moore laboratory revealed that TaZIP4-B2 promotes homologous bivalent formation by preventing recombination intermediates established between homoeologous chromosomes from becoming crossovers $^{17,56}$. In contrast to MMR proteins, there is no indication of ZIP4 involvement in promoting heteroduplex rejection following DNA-strand exchange between divergent sequences. This thus suggests that TaMSH7-3D and TaZIP4-B2 could act sequentially with different modes of action and consequently that homoeologous recombination is controlled by a multilayered mechanism in polyploid bread wheat. ph1 was found to be twice as strong as $p h 2^{44}$ and an additive effect of these mutations in promoting homoeologous recombination has been reported, for example in wheat/Aegilops hybrids ${ }^{33}$. Combining Tazip4-B2 and Tamsh7-3D mutations may therefore offer an opportunity to further improve the efficiency and ease of introgression of wild relative chromosomal segments into wheat, providing opportunities for the development of genetically unique and desirable wheat varieties. Exploitation of Tazip4-B2 and Tamsh7-3D EMSderived double mutants that are in the elite background Cadenza, are likely to be of particular interest to pre-breeders compared to previously available Chinese Spring mutants ( $p h 1 b, p h 2 a)$, as time required to move an introgression into a breeding relevant genotype is reduced. Additionally, the utilisation of point mutations is likely to avoid possible meiotic instability that can be induced by large chromosomal deletions.

TaMSH7-3D has two highly similar homoeologous copies on chromosomes $3 \mathrm{~A}$ and 3B, TaMSH7-3A and TaMSH7-3B, with which it shares $97.77 \%$ and $97.96 \%$ identity, respectively (Supplementary Table 9). Because of possible functional redundancy and their genomic locality, it is reasonable to assume that TaMSH7-3A and TaMSH7-3B could correspond to the homoeologous recombination suppressors previously identified on $3 \mathrm{AS}^{35,57}$ and $3 \mathrm{BS}^{58}$. This also takes into consideration that the loss of both $3 \mathrm{AS}$ and 3DS (Ph2) was observed to result in a level of chiasma frequency between homoeologous chromosomes similar to that caused by the deficiency of $5 \mathrm{~B}(P h 1)^{35}$. An interesting question is what could be the cause for differences in phenotypic severity observed between homoeologues (3DS $>3$ AS $>3 \mathrm{BS})^{58}$ ? As TaMSH7-3A, TaMSH7-3B and TaMSH7-3D show comparable RNA abundance in wheat anthers during early meiosis (Fig. 4), a difference derived from transcriptional level is unlikely. Although TaMSH7-3A, TaMSH7-3B and TaMSH7-3D proteins are very similar ( $>96.3 \%$ of sequence identity), mutation prediction algorithms have hinted to potentially deleterious amino acid substitutions between homoeologues (e.g. L877S in TaMSH7-3B and R855H in TaMSH7-3A). However, these predictions are indicative and require experimental validation. Additionally, a shared 28 bp deletion predicted to lead to a nonfunctional TaMSH7-3A protein in 14 wheat related accessions (Supplementary Figs. 9 and S10, Supplementary Table 11) 
indicates that TaMSH7-3A has degenerated into a pseudogene. This could potentially reflect progressive duplicated gene loss which is particularly rapid for meiotic genes - following polyploidization events described in Angiosperms ${ }^{59}$. Generation of CRISPR/Cas9 mutants for one or more TaMSH7 copies will allow confirmation of their relative impact on homoeologous recombination as well as determine their combinatorial effects, opening an opportunity to further refine exotic chromatin introgression into elite wheats.

Taking advantage of newly available genetic and bioinformatic resources, this research has answered a 50-year-old question on the causative agent for Ph2 by identifying TaMSH7-3D. This work provides fundamental insights into the molecular control of meiotic recombination in allopolyploids and opens a path towards more efficient and flexible access to genetic diversity, a major bottleneck currently facing crop improvement.

\section{Methods}

Plant material and growth conditions. Plant material used in this study included the following: wild-type hexaploid wheat (Triticum aestivum cv. Chinese Spring and cv. Cadenza); Chinese Spring ph $2 a$ and ph $2 b$ mutants; 32 Chinese Spring 3Ddeletion lines (from Svačina et al. ${ }^{23}$ ) and four Cadenza Tamsh7-3D mutant lines (Tamsh7-3D A467T (Cadenza0638); Tamsh7-3D D642N (Cadenza1114); Tamsh73D G463R (Cadenza1178); Tamsh7-3D Q605* (Cadenza2006) (Supplementary Table 3; www.wheat-tilling.com)). Plants were genotyped by PCR amplification using primers specific to wild-type or EMS mutant alleles (Supplementary Table 13). Wild-type cv. Chinese Spring, ph2a, ph $2 b$ and the 3D-deletion lines were crossed with rye (Secale cereale; $\mathrm{RR}, 2 n=14$; var. Dankowski nove) to produce wheat/rye haploid hybrids (ABDR, $n=28$ ). Wild-type cv. Cadenza and the Tamsh7-3D mutant lines were crossed with Aegilops variabilis (UUSS, $2 n=4 x=$ 28 ) to produce wheat/Ae. variabilis haploid hybrids (ABDUS, $n=35$ ). Plants were grown in a controlled-environment room with the following conditions: $16 \mathrm{~h}$ light/ $8 \mathrm{~h}$ night photoperiod at $20^{\circ} \mathrm{C}$ day and $15^{\circ} \mathrm{C}$ night, with $70 \%$ humidity.

Cytological analysis. Young spikes were collected from 7 to 10 -week-old plants and carefully dissected to isolate anthers. For each dissected floret, one of the three developmentally equivalent anthers was squashed in aceto-carmine staining solution and meiocytes visualised using a ZEISS Optima microscope. When meiocytes at metaphase I were identified (for chiasma frequency analysis) or other defined stages (RNA analysis), the two remaining anthers were either fixed in $100 \%$ ethanol/acetic acid 3:1 (v/v) for $48 \mathrm{~h}$ and then subsequently transferred to $70 \%$ ethanol or snap frozen in liquid $\mathrm{N}_{2}$ for later RNA-based analyses. Fixed anthers can eventually be stored at $4^{\circ} \mathrm{C}$ for a few months. For cytological analysis of meiocytes at metaphase I, pollen mother cells (PMCs) were released from the anther by crushing it on a slide in a drop of aceto-carmine staining solution. Anther debris was carefully removed, and a coverslip placed on the slide. The slides were then heated until separation of the chromosomes and aceto-carmine solution replaced by acetic acid $45 \%$. Coverslips were then vertically pressed to spread out the chromosomes. Chromosome configurations of $\sim 50$ PMC per anther were analysed under a ZEISS Axio Observer Z1 inverted microscope. For each cell, the number of univalents, rod bivalents (pair of chromosomes linked by a unique chiasma), ring bivalents (pair of chromosomes linked by two chiasmata), trivalents (three chromosomes linked by two chiasmata) and quadrivalents (four chromosomes linked by three or four chiasmata) were counted. Frequency of chiasmata (the cytological manifestation of meiotic crossovers) was then calculated. Significant differences between mutant and corresponding wild-type control chiasma frequencies were assessed using Mann-Whitney tests adjusted for multiple comparisons.

Exome capture and bioinformatic analysis. DNA was extracted from leaf material (10 cm long young, fresh and healthy leaves) from wild-type bread wheat cv. Chinese Spring, ph $2 a$ and $p h 2 b$ by first snap-freezing the tissue in liquid nitrogen and crushing it into a fine powder (using knitting needle). The powder was resuspended in $600 \mu \mathrm{L}$ of extraction buffer $(0.1 \mathrm{M}$ TRIS- $\mathrm{HCl} \mathrm{pH} 8,10 \mathrm{mM}$ EDTA, $0.1 \mathrm{M} \mathrm{NaCl}, 1 \%$ sarkosyl, 2\% polyvinyl-poplypyrrolidone (insoluble) and followed by addition of $600 \mu \mathrm{L}$ phenol-chloroform-isoamyl alcohol (25:24:1) and hand mixing. The phases were separated by centrifugation in an Eppendorf centrifuge and the aqueous phase was re-extracted. After the second phenolchloroform-isoamyl alcohol extraction, the aqueous phase was extracted once with an equal volume of chloroform. DNA was precipitated by the addition of $0.1 \mathrm{vol}$ of $3 \mathrm{M}$ sodium acetate ( $\mathrm{pH} 4.8$ ) and $1 \mathrm{vol}$ of propan-2-ol. The DNA pellet was washed with $70 \%$ ethanol and resuspended in $30 \mu \mathrm{L}$ TE buffer [10 mM TRIS-HC1 (pH 8.0), $1 \mathrm{mM}$ EDTA] containing $40 \mu \mathrm{g} / \mathrm{ml}$ RNase A.

Purified DNA samples were sent to Arbor Biosciences (USA) for whole exome capture and sequencing. Genomic DNA was sonicated using a Q800R sonicator
(Qsonica, CT, USA) and size-selected using SPRI beads to modal insert lengths of roughly $400 \mathrm{bp}$. Then $200 \mathrm{ng}$ of the resulting processed DNA was converted to Illumina Truseq-style libraries using in-house chemistry and six cycles of dual-8bpbarcode indexing amplification. Target enrichment reactions were performed in singleplex using $750 \mathrm{ng}$ of each library and the Arbor Wheat Exome Beta probe set The enrichment procedure followed the standard myBaits version 4.0 manual (https://arborbiosci.com/wp-content/uploads/2018/04/myBaits-Manual-v4.pdf), but with $0.75 \mu \mathrm{L}$ IDT xGen Universal Blocking Oligos (Integrated DNA Technologies, USA) in lieu of $0.5 \mu \mathrm{L}$ Block A. Following capture clean-up as described in the myBaits user manual, libraries were submitted for $150 \mathrm{bp}$ pairedend sequencing on a partial NovaSeq S4 lane (Illumina). Following sample demultiplexing using both barcodes per library, read pairs were taken to bioinformatic analysis.

Read qualities were inspected with FASTQC version $0.11 .4^{60}$ before and after quality and adapter trimming. For the exome capture data, trimming was achieved with Trimmomatic version $0.36^{61}$, using the following parameters: -phred 33 LEADING:5 TRAILING:5 SLIDINGWINDOW:4:20 MINLEN:50.

Cleaned exome capture reads were aligned to the CS Ref v1.0 by Bowtie2 version $2.3 .0^{62}$ allowing a $2 \%$ mismatch rate with the following parameters: --endto-end --very-sensitive --n-ceil L,0,0.1 --rdg 3,3 --rfg 3,3 --no-unal --mp 6,6 --np 4 --no-mixed --score-min L,0,-0.12. After alignment, PCR duplicates were detected and removed from bam files using an in-house Java application Wheatbio.jar (https://github.com/CroBiAd/TILLinG-mutants).

After read alignment, a pileup file was generated from the bam files of the exome capture data using the mpileup command (-min-MQ $20-\mathrm{B}-\mathrm{f})$ in samtools version $1.4 .1^{63}$. The pileup file was subsequently used to identify genome-wide SNPs and indels. To determine the effect of the detected polymorphisms the CS Ref v1.0 annotation, specifically the high-confidence gene models, was relied upon. To avoid calling false positive polymorphisms, we demanded that each SNP or indel position was supported by a read depth of $\geq 4$. To predict the consequences of mutations on protein sequences, we used SNPeff version $4.3^{64}$ and the annotation from CS Ref v1.0 choosing the longest predicted splice-form.

RNA sequencing and processing. Anthers cytologically determined to be at metaphase I or earlier were pooled for RNA extraction according to Tucker et al. ${ }^{65}$ These purified early-meiotic anther RNA samples derived from $p h 2 b$ and Chinese Spring were submitted to the Australian Genome Research Facility (AGRF, Australia) for library preparation and sequencing on the Illumina NovaSeq 6000 instrument. Stranded cDNA was generated from poly-adenylated RNA by TruSeq stranded mRNA library kits (Illumina). Samples were sequenced to give $150 \mathrm{bp}$ paired-end reads aiming at around 130 Mill reads/sample.

RNASeq raw data was processed with fastp version $0.19 .7^{66}$ within minimum length requirement of 60 , trimming of poly $\mathrm{G}$ and removal of the first 10 bases. After trimming we had 144,818,345 clean paired-end reads for $p h 2 b$ replicate 1 and $141,336,841$ reads for ph $2 b$ replicate 2 . For the wild-type Chinese Spring we obtained 130,912,185 and 135,248,954 reads for the two replicates, respectively. Trimmed RNASeq reads were aligned using STAR version $2.5 .3^{67}$ to CS Ref v1.0 with the following parameters: --outFilterMismatchNoverLmax 0.02 --outFilterMatchNminOverLread 0.98 --outFilterMultimapNmax 5 --outFilterMultimapScoreRange 0 --outFilterScoreMinOverLread 0 --alignEndsType Local --alignIntronMax 10000 --alignMatesGapMax 10500 --alignSoftClipAtReferenceEnds No --outSJfilterOverhangMin 35202020 --outSJfilterCountTotalMin 10333 --outSJfilterCountUniqueMin 51111 .

Genetic diversity and phylogenetic analysis. The MSH7-3A and 3B sequences were used as queries in BLASTN searches against available genomic sequence data generated by the Wheat Initiative's $10+$ Genomes project (https://webblast.ipkgatersleben.de/wheat_ten_genomes/) and for the UK varieties https://wheatis. earlham.ac.uk//grassroots-portal/blast. Australian varieties were inspected for variation in MSH7-3A and 3B in DAWN (http://crobiad.agwine.adelaide.edu.au/ dawn/jbrowse/). Sequences for Cadenza and Paragon were retrieved and trimmed to exon2 for CLUSTALW alignment. Public databases were searched for the presence of the 28 bp deletion by either using the full MSH7-3A sequence or for NCBI-SRA data with a 300 bp subsequence covering the deleted region. In addition we queried DAWN ${ }^{42}$ at position chr3A:87373944..87374105 for the presence of the deletion in Bioplatforms Australia sequenced varieties (https://data.bioplatforms. com/organization/bpa-wheat-cultivars). Pedigree information was retrieved from GRIS (http://wheatpedigree.net/) when available or the literature otherwise.

Grass sequences homologous to MSH7 were identified by BLAST searches against a range of databases (Supplementary Table 12). Whenever gene models were non-existent or incomplete, putative homologs were manually derived from genomic DNA, and their protein sequences deduced.

DNA and protein sequences were aligned by MUSCLE in Geneious version 10 and percentage identities calculated. The unrooted phylogenetic tree was inferred using PhyML v20160115 ran with model JTT and parameters: --nclasses 4 -f m -alpha e -pinv e -bootstrap $100-\mathrm{o} \operatorname{tlr}^{68}$. Branch supports are computed out of 100 bootstrapped trees. 
Reporting summary. Further information on research design is available in the Nature Research Reporting Summary linked to this article.

\section{Data availability}

Data supporting the findings of this work are available within the paper and its Supplementary Information files. A reporting summary for this article is available as a Supplementary Information file. The datasets and plant materials generated and analysed during the current study are available from the corresponding author upon request. Exome capture and RNA Seq data have been deposited in the NCBI SRA database under BioProject PRJNA648242. Exome capture data have accession numbers SRR12315376 and SRR12315377, RNAseq data are under SRR13364311 and SRR13364312. The genomic sequences of TaMSH7-3D from ph $2 b$ mutant and TaMSH7-3A from Cadenza are available in Supplementary Data 1. Source data are provided with this paper.

\section{Code availability}

Code is available at GitHub [https://github.com/CroBiAd/TILLinG-mutants].

Received: 29 July 2020; Accepted: 13 January 2021;

Published online: 05 February 2021

\section{References}

1. Hajjar, R. \& Hodgkin, T. The use of wild relatives in crop improvement: a survey of developments over the last 20 years. Euphytica 156, 1-13 (2007).

2. Mercier, R., Mézard, C., Jenczewski, E., Macaisne, N. \& Grelon, M. The molecular biology of meiosis in plants. Annu. Rev. Plant Biol. 66, 297-327 (2015).

3. Wood, T. E. et al. The frequency of polyploid speciation in vascular plants. Proc. Natl Acad. Sci. 106, 13875-13879 (2009).

4. Blake, N. K., Lehfeldt, B. R., Lavin, M. \& Talbert, L. E. Phylogenetic reconstruction based on low copy DNA sequence data in an allopolyploid: the B genome of wheat. Genome 42, 351-360 (1999).

5. Huang, S. et al. Genes encoding plastid acetyl-CoA carboxylase and 3phosphoglycerate kinase of the Triticum/Aegilops complex and the evolutionary history of polyploid wheat. Proc. Natl Acad. Sci. 99, 8133-8138 (2002).

6. Riley, R. \& Chapman, V. Genetic control of the cytologically diploid behaviour of hexaploid wheat. Nature 182, 713-715 (1958).

7. Sears, E. R. Intergenomic chromosome relationships in hexaploid wheat. Proc. Int. Congr. Genet. 2, 258-259 (1958).

8. Mello-Sampayo, T. \& Lorente, R. The role of chromosome 3D in the regulation of meiotic pairing in hexaploid wheat. EWAC Newsl. 2, 16-24 (1968).

9. Mello-Sampayo, T. Genetic regulation of meiotic chromosome pairing by chromosome 3D of Triticum aestivum. Nat. N. Biol. 230, 22-23 (1971).

10. Giorgi, B. \& Barbera, F. Use of mutants that affect homoeologous pairing for introducing alien variation in both durum and common wheat. (International Atomic Energy Agency, 1981).

11. Giorgi, B. \& Barbera, F. Increase of homoeologous pairing in hybrids between a ph mutant of T. turgidum L. var. durum and two tetraploid species of Aegilops: Aegilops kotschyi and Ae. cylindrica. Cereal Res. Commun. 9, 205-2 (1981).

12. Sears, E. R. An induced mutant with homoeologous pairing in wheat. Can. J. Genet. Cytol. 19, 585-593 (1977).

13. Gill, K. S. \& Gill, B. S. A DNA fragment mapped within the submicroscopic deletion of $\mathrm{Ph} 1$, a chromosome pairing regulator gene in polyploid wheat. Genetics 129, 257-259 (1991).

14. Gill, K. S., Gill, B. S., Endo, T. R. \& Mukai, Y. Fine physical mapping of Ph1, a chromosome pairing regulator gene in polyploid wheat. Genetics 134, 1231-1236 (1993).

15. Griffiths, S. et al. Molecular characterization of $\mathrm{Ph} 1$ as a major chromosome pairing locus in polyploid wheat. Nature 439, 749-752 (2006).

16. Al-Kaff, N. et al. Detailed dissection of the chromosomal region containing the Ph1 locus in wheat Triticum aestivum: with deletion mutants and expression profiling. Ann. Bot. 101, 863-872 (2008).

17. Martín, A. C., Rey, M.-D., Shaw, P. \& Moore, G. Dual effect of the wheat Ph1 locus on chromosome synapsis and crossover. Chromosoma 126, 669-680 (2017).

18. Sidhu, G. K., Rustgi, S., Shafqat, M. N., von Wettstein, D. \& Gill, K. S. Fine structure mapping of a gene-rich region of wheat carrying $\mathrm{Ph} 1$, a suppressor of crossing over between homoeologous chromosomes. Proc. Natl Acad. Sci. 105, 5815-5820 (2008)
19. De Muyt, A. et al. A meiotic XPF-ERCC1-like complex recognizes joint molecule recombination intermediates to promote crossover formation. Genes Dev. 32, 283-296 (2018).

20. Rey, M.-D. et al. Exploiting the ZIP4 homologue within the wheat Ph1 locus has identified two lines exhibiting homoeologous crossover in wheat-wild relative hybrids. Mol. Breed. 37, 95 (2017).

21. Rey, M. D. et al. Magnesium increases homoeologous crossover frequency during meiosis in ZIP4 (Ph1 gene) mutant wheat-wild relative hybrids. Front. Plant Sci. 9, 1-12 (2018).

22. Sutton, T. et al. The Ph 2 pairing homoeologous locus of wheat (Triticum aestivum): identification of candidate meiotic genes using a comparative genetics approach. Plant J. 36, 443-456 (2003).

23. Svačina, R. et al. Development of deletion lines for chromosome $3 \mathrm{D}$ of bread wheat. Front. Plant Sci. 10, 1-6 (2020).

24. Ji, L.-H. \& Langridge, P. An early meiosis cDNA clone from wheat.Mol. Gen. Genet. 243, 17-23 (1994)

25. Whitford, R. From Intimate Chromosome Associations to Wild Sex in Wheat (Triticum aestivum). (University of Adelaide, Australia, 2002).

26. Letarte, J. Identification and Characterisation of Early Meiotic Genes in Wheat (University of Adelaide, Australia, 1996).

27. Dong, C. et al. WM5: isolation and characterisation of a gene expressed during early meiosis and shoot meristem development in wheat. Funct. Plant Biol. 32, 249 (2005).

28. Dong, C., Whitford, R. \& Langridge, P. A DNA mismatch repair gene links to the $\mathrm{Ph} 2$ locus in wheat. Genome 45, 116-124 (2002).

29. Lloyd, A. H., Milligan, A. S., Langridge, P. \& Able, J. A. TaMSH7: a cereal mismatch repair gene that affects fertility in transgenic barley (Hordeum vulgare L.). BMC Plant Biol. 7, 1-9 (2007).

30. Wall, A. M., Riley, R. \& Chapman, V. Wheat mutants permitting homoeologous meiotic chromosome pairing. Genet. Res. 18, 311-328 (1971).

31. Sears, E. R. A wheat mutation conditioning an intermediate level of homoeologous chromosome pairing. Can. J. Genet. Cytol. 24, 715-719 (1982)

32. Martinez, M., Cuñado, N., Carcelén, N. \& Romero, C. The Ph1 and Ph2 loci play different roles in the synaptic behaviour of hexaploid wheat Triticum aestivum. Theor. Appl. Genet. 103, 398-405 (2001).

33. Ceoloni, C. \& Donini, P. Combining mutations for the two homoeologous pairing suppressor genes $\mathrm{Ph} 1$ and $\mathrm{Ph} 2$ in common wheat and in hybrids with alien Triticeae. Genome 36, 377-386 (1993).

34. Appels, R. et al. Shifting the limits in wheat research and breeding using a fully annotated reference genome. Science 361, 6403 (2018).

35. Mello-Sampayo, T. \& Canas, P. Suppressors of meiotic chromosome pairing in common wheat. Proc 4th Wheat Genet. Symp. Columbia, Missouri, USA 709-713 (1973).

36. King, R. et al. Mutation scanning in wheat by exon capture and nextgeneration sequencing. PLoS One 10, e0137549 (2015).

37. Krasileva, K. V. et al. Uncovering hidden variation in polyploid wheat. Proc Natl Acad. Sci. 114, 913-921 (2017).

38. Tam, S. M., Hays, J. B. \& Chetelat, R. T. Effects of suppressing the DNA mismatch repair system on homeologous recombination in tomato. Theor. Appl. Genet. 123, 1445-1458 (2011).

39. Chirinos-arias, M. C. \& Spampinato, C. P. Plant physiology and biochemistry growth and development of AtMSH7 mutants in Arabidopsis thaliana. Plant Physiol. Biochem. 146, 329-336 (2020).

40. Alabdullah, A. K. et al. A co-expression network in hexaploid wheat reveals mostly balanced expression and lack of significant gene loss of homeologous meiotic genes upon polyploidization. Front. Plant Sci. 10, 1325 (2019)

41. Martín, A. C. et al. Genome-wide transcription during early wheat meiosis is independent of synapsis, ploidy level, and the Ph1 locus. Front. Plant Sci. 9, 1-19 (2018)

42. Watson-Haigh, N. S., Suchecki, R., Kalashyan, E., Garcia, M. \& Baumann, U. DAWN: a resource for yielding insights into the diversity among wheat genomes. BMC Genom. 19, 1-20 (2018).

43. Sears, E. R. Homoeologous chromosomes in Triticum aestivum. Genetics 37, 624 (1952).

44. Sears, E. R. Genetic control of chromosome pairing in wheat. Annu. Rev. Genet. 10, 31-51 (1976)

45. Reyes, G. X. et al. New insights into the mechanism of DNA mismatch repair Chromosoma 124, 443-462 (2016).

46. Culligan, K. M. \& Hays, J. B. Arabidopsis MutS homologs - AtMSH2, AtMSH3, AtMSH6, and a Novel AtMSH7 - form three distinct protein heterodimers with different specificities for mismatched DNA. Plant Cell 12, 991-1002 (2000).

47. Wu, S. Y., Culligan, K., Lamers, M. \& Hays, J. Dissimilar mispair-recognition spectra of Arabidopsis DNA-mismatch-repair proteins MSH2.MSH6 (MutSa) and MSH2.MSH7 (MutSץ). Nucleic Acids Res. 31, 6027-6034 (2003).

48. Gómez, R. \& Spampinato, C. P. Mismatch recognition function of Arabidopsis thaliana MutSy. DNA Repair 12, 257-264 (2013). 
49. McCulloch, S. D., Gu, L. \& Li, G. M. Bi-directional processing of DNA loops by mismatch repair-dependent and -independent pathways in human cells. $J$. Biol. Chem. 278, 3891-3896 (2003).

50. Tian, L., Gu, L. \& Li, G. M. Distinct nucleotide binding/hydrolysis properties and molar ratio of MutS $\alpha$ and MutS $\beta$ determine their differential mismatch binding activities. J. Biol. Chem. 284, 11557-11562 (2009).

51. Culligan, K. M. Evolutionary origin, diversification and specialization of eukaryotic MutS homolog mismatch repair proteins. Nucleic Acids Res. 28, 463-471 (2000).

52. Lario, L. D., Botta, P., Casati, P. \& Spampinato, C. P. Role of AtMSH7 in UVB-induced DNA damage recognition and recombination. J. Exp. Bot. 66, 3019-3026 (2015).

53. Chakraborty, U. \& Alani, E. Understanding how mismatch repair proteins participate in the repair/anti-recombination decision. FEMS Yeast Res. 16, 1-12 (2016).

54. $\mathrm{Hu}, \mathrm{Q}$. et al. Meiotic chromosome association 1 interacts with TOP $3 a$ and regulates meiotic recombination in rice. Plant Cell 29, 1697-1708 (2017).

55. Fernandes, J. B. et al. FIGL1 and its novel partner FLIP form a conserved complex that regulates homologous recombination. PLoS Genet. 14, e1007317 (2018).

56. Martín, A. C., Shaw, P., Phillips, D., Reader, S. \& Moore, G. Licensing MLH1 sites for crossover during meiosis. Nat. Commun. 5, 4580 (2014)

57. Driscoll, C. J. Genetic suppression of homoeologous chromosome pairing in hexaploid wheat. Can. J. Genet. Cytol. 14, 39-42 (1972).

58. Miller, T. E., Reader, S. M. \& Gale, M. D. The effect of homoeologous group 3 chromosomes on chromosome pairing and crossability in Triticum aestivum. Can. J. Genet. Cytol. 25, 634-641 (1983).

59. Lloyd, A. H. et al. Meiotic gene evolution: can you teach a new dog new tricks? Mol. Biol. Evol. 31, 1724-1727 (2014).

60. Andrews, S. FastQC: a quality control tool for high throughput sequence data. Babraham Bioinforma. (2016). https://www.bioinformatics.babraham.ac.uk/ projects/fastqc/.

61. Bolger, A. M., Lohse, M. \& Usadel, B. Trimmomatic: a flexible trimmer for Illumina sequence data. Bioinformatics 30, 2114-2120 (2014).

62. Langmead, B. \& Salzberg, S. L. Fast gapped-read alignment with Bowtie 2. Nat. Methods 9, 357-359 (2012)

63. Li, H. et al. The sequence alignment/map format and SAMtools. Bioinformatics 25, 2078-2079 (2009).

64. Cingolani, P. et al. A program for annotating and predicting the effects of single nucleotide polymorphisms, SnpEff. Fly. 6, 80-92 (2012).

65. Tucker, E. J. et al. Molecular identification of the wheat male fertility gene Ms1 and its prospects for hybrid breeding. Nat. Commun. 8, 869 (2017).

66. Chen, S., Zhou, Y., Chen, Y. \& Gu, J. Fastp: an ultra-fast all-in-one FASTQ preprocessor. Bioinformatics 34, i884-i890 (2018).

67. Dobin, A. et al. STAR: ultrafast universal RNA-seq aligner. Bioinformatics 29, 15-21 (2013)

68. Guindon, S. et al. New algorithms and methods to estimate maximumlikelihood phylogenies: assessing the performance of PhyML 3.0. Syst. Biol. 59, 307-321 (2010).

69. Zadoks, J. C., Chang, T. T. \& Konzak, C. F. A decimal code for the growth stages of cereals. Weed Res. 14, 415-421 (1974).

70. Pingault, L. et al. Deep transcriptome sequencing provides new insights into the structural and functional organization of the wheat genome. Genome Biol. 16, 1-15 (2015).

\section{Acknowledgements}

We warmly acknowledge Isabelle Lhommet and Amelie Jeanneau for technical support and Hélène Rimbert, Wandrille Duchemin and Jonathan Kitt for bioinformatics support We thank Peter Langridge (University of Adelaide) for providing the ph2a and ph2b mutants, and for the support provided to the Australian authors over many years of meiosis research in his laboratory. We also thank Eric Jenczewski (INRAE, IJPB, Versailles) for critical reading of the manuscript and helpful discussion. This work was supported by EU-funded AgreenSkills+ fellowship, ANR CROC (CE19-2014), the Bettencourt-Schueller Foundation, the Czech Science Foundation (grant award 17-05341 $S)$ and the ERDF project "Plants as a tool for sustainable global development" (CZ.02.1.01/0.0/0.0/16_019/0000827).

\section{Author contributions}

H.S., R.S., U.B., R.W., T.S., J.B. and P.S. conceived and designed the experiments. H.S., R.S. and U.B. performed the experiments. H.S., R.S., U.B., R.W. and P.S. analysed the data. H.S. and R.W. wrote the manuscript with inputs from U.B. and P.S. All authors approved the manuscript.

\section{Competing interests}

The authors declare no competing interests.

\section{Additional information}

Supplementary information The online version contains supplementary material available at https://doi.org/10.1038/s41467-021-21127-1.

Correspondence and requests for materials should be addressed to H.S. or P.S.

Peer review information Nature Communications thanks the anonymous reviewers for their contribution to the peer review of this work. Peer reviewer reports are available.

Reprints and permission information is available at http://www.nature.com/reprints

Publisher's note Springer Nature remains neutral with regard to jurisdictional claims in published maps and institutional affiliations.

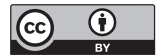

Open Access This article is licensed under a Creative Commons Attribution 4.0 International License, which permits use, sharing, adaptation, distribution and reproduction in any medium or format, as long as you give appropriate credit to the original author(s) and the source, provide a link to the Creative Commons license, and indicate if changes were made. The images or other third party material in this article are included in the article's Creative Commons license, unless indicated otherwise in a credit line to the material. If material is not included in the article's Creative Commons license and your intended use is not permitted by statutory regulation or exceeds the permitted use, you will need to obtain permission directly from the copyright holder. To view a copy of this license, visit http://creativecommons.org/ licenses/by/4.0/

(C) The Author(s) 2021 